\title{
Computation of Bipolar Transistor Base Parameters for General Distribution of Impurities in Base
}

\author{
AVRAHAM GOVER, JAN GRINBERG, AND ADY SEIDMAN
}

\begin{abstract}
A procedure is suggested by which dc and ac base gain parameters can be computed for general impurity distributions in the base. The procedure consists of solving the current equation as series in the recombination time $(1 / \tau)$.

The series expansion coefficients are computed for a Gaussian distribution up to first order, along with the resulting base alpha transport factor and the transit time. Mobility variation with impurity concentration is also taken into account.

Explicit expressions for cutoff frequencies and excess phase shift $\left(\omega_{T}, \omega_{\alpha}, \omega_{\beta}, m\right)$ are developed using the coefficients of the series expansion up to the second order. Computation of these parameters for the case of an exponential distribution, with and without assumption of diffusion coefficient variation, results in new expressions and values.
\end{abstract}

\section{Definition of Symbols}

$N_{A}(x)$ Acceptors concentration.

$N_{S} \quad$ Surface concentration of acceptor impurities profile.

$N_{A_{E B}}$ Acceptor impurities concentration at emitter-base junction.

$x_{1} \quad$ Emitter-base junction depth.

$x_{2} \quad$ Base-collector junction depth.

$W \quad$ Base width.

$n^{\prime}(x) \quad$ Excess electrons concentration.

$n_{1}^{\prime} \quad$ Equivalent to $n^{\prime}\left(x_{1}\right)$.

$J_{n}{ }^{(0)} \quad$ Current density of injected electrons.

$\alpha_{T^{\prime}} \quad$ Base transport factor.

$m$ Excess phase shift.

$\omega_{\alpha} \quad$ Cutoff frequency for $\alpha$.

$\omega_{\beta} \quad$ Cutoff frequency for $\beta$.

$\omega_{T} \quad$ Gain-bandwidth product.

\section{INTRODUCTION}

$\$$ INCE the introduction of drift transistors into widespread production, many authors [2], [3], [7], [8] have tried to fit a theory to the nonhomogeneous base distribution. The nonhomogeneous base problem is more complicated than the homogeneous one, not only in the additional field term, but also in having the diffusion coefficient varying across the base.

One possible approach to the solution of the nonhomogeneous base problem [2], [8] consists of keeping the

Manuscript received July 7, 1971; revised March 9, 1972.

A. Gover was with the Department of Physics and Astronomy Tel-Aviv University, Tel-Aviv, Israel. He is now at the Department of Applied Physics, California Institute of Technology, Pasadena, Calif.

J. Grinberg and A. Seidman are with the Department of Physics and Astronomy, Tel-Aviv University, Tel-Aviv, Israel.

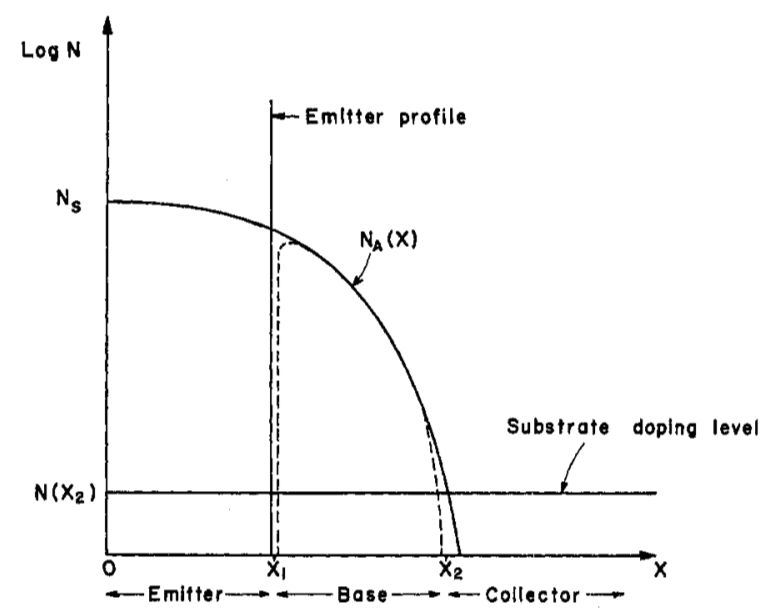

Fig. 1. Illustration of one-dimensional impurity distribution in transistor. (For more accurate analysis one may choose to define $x_{1}$ and $x_{2}$ at the edges of the emitter-base and base-collector depletion regions, respectively. The following derivations apply equally for this choice.)

convenient gain parameters of basic theory [1], [4] such as $\alpha_{T}, m, \omega_{\alpha}, \omega_{\beta}, \omega_{T}$ (not all independent of each other!). A recently published paper [9] best presents the other approach, consisting of an exact computer calculation of $\alpha(\omega)$ for specified impurity distributions (in [9] the Craussian and error functions were taken as examples, with the origin at the emitter-base junction). This approach, although accurate, has the disadvantage of giving an exact solution only for each individual case, i.e., an entire calculation must be done not only for each separate impurity distribution, but also for each individual distribution parameter.

The approach of this paper is basically the first one. We develop a mathematical procedure to solve the excess minority carrier equation for a general impurity distribution and any assumption of mobility variation, yielding explicit expressions for the above-mentioned gain parameters $\left(\alpha_{T}, m, \omega_{\alpha}, \omega_{\beta}, \omega_{T}\right)$ in the same general conditions (considering base transport contribution only).

The examples that are treated using this procedure were chosen with special reference to the commonly used double-diffusion production process for silicon transistors. Therefore, the base impurity distribution is assumed to be either exponential or Gaussian, with the origin lying at the transistor surface (Fig. 1), and the diffusion coefficient is assumed to be either constant or power-law dependent on the impurity distribution [5]. 
All derivations and calculations are done for $n-p-n$ transistors, but the same results apply also for $\mathrm{p}-\mathrm{n}-\mathrm{p}$ transistors with an appropriate change of symbols.

\section{Base DC Parameters Calculation}

We intend to calculate the current and excess minority carriers in the base in order to derive base electrical parameters.

We assume that the excess minority carriers $n^{\prime}$ and the minority carriers current density $J_{n}$ are governed by the equations

$$
\begin{aligned}
& J_{n}=q D_{n} \cdot \frac{d n^{\prime}}{d x}+q \mu_{n} n^{\prime} \cdot E \\
& \frac{n^{\prime}}{\tau_{n}}=\frac{1}{q} \frac{d J_{n}}{d x}
\end{aligned}
$$

but, since the two terms of $J_{n}$, without injection, cancel each other, and assuming low-level injection, so that $E$, the built-in field resulting from the gradient of the specified base impurity distribution [2]

$$
E=-\frac{d \psi}{d x}=\frac{k T}{q} \frac{1}{N_{A}} \frac{d N_{A}}{d x}
$$

is also true for excess minority carriers $n^{\prime}$.

The boundary conditions for (1) and (2) for conditior of short-circuited collector junction are

$$
\begin{aligned}
n^{\prime}\left(x_{1}\right) & \equiv n_{1}^{\prime}=n_{p}\left(x_{1}\right) \cdot\left(e^{q V / k T}-1\right) \\
n^{\prime}\left(x_{2}\right) & =0
\end{aligned}
$$

where $x_{1}$ and $x_{2}$ are emitter-base and base-collecto: junctions, respectively. These boundary conditions alsi hold approximately for normal biasing in the activ? region.

Alternatively, instead of (4) we can require the bound. ary condition

$$
J_{n}\left(x_{1}\right)=J_{n}{ }^{(0)} .
$$

Equations (1)-(3) together with boundary conditions (4) and (5), or (6) and (5), form a well-defined matheme. tical problem. The simplest formal method of solution is to substitute one in to the other and solve the resulting. second-order differential equation with boundary conditions. This has actually been done for a homogeneous base [1] and for an exponential impurity distribution in the base [2], where immediately integrable differential equations were obtained. Unfortunately, for other pra:tical impurity distributions, the resulting differential equation is not analytically integrable. Fortunately, as $\left(W / L_{n}\right)^{2}$ is very small, a first-order solution for dc parameters is generally sufficient. Therefore, althoug, h a solution for the homogeneous base has been found [1], expansion to first order in recombination $(1 / \tau)$ is cor 7 monly used:

$$
\begin{aligned}
& \alpha_{T}=1-\frac{1}{2} \cdot \frac{W^{2}}{L_{n}^{2}} \\
& L_{n}=\left(D_{n} \cdot \tau_{n}\right)^{\frac{1}{2}} .
\end{aligned}
$$

Following expression (7), we will present a straightforward procedure for calculating the terms of series ex. pansion of $\alpha_{T}$ in the case of a general impurity distribu-. tion. For dc conditions, the first-order term will be sufficient. We will show later that for ac conditions, expansion up to second order is sufficient:

$$
\alpha_{T} \equiv 1-u_{1} \cdot \frac{W^{2}}{L_{n}{ }^{2}}+u_{2} \cdot \frac{W^{4}}{L_{n}{ }^{4}}-\cdots
$$

Substituting (3) into (1) and using the Einstein relation,

$$
J_{n}(x)=q D_{n} \frac{1}{N_{A}} \frac{d}{d x}\left(n^{\prime} \cdot n_{A}\right) .
$$

We transform (10) and (2) into integral form, implicitly including the boundary conditions (5) and (6): 1

$$
\begin{aligned}
& n^{\prime}(x)=-\frac{1}{q N_{A}} \int_{x}^{x_{2}} \frac{N_{A}}{D_{n}} \cdot J_{n} d x \\
& J_{n}(x)=J_{n}{ }^{(0)}+\frac{q}{\tau_{n}} \int_{x_{1}}^{x} n^{\prime} d x .
\end{aligned}
$$

Now $n^{\prime}(x)$ and $J_{n}(x)$ can be calculated as series in recombination:

$$
\begin{aligned}
J_{n}(x) & =J_{n}{ }^{(0)}(x)+J_{n}^{(1)}(x)+\cdots \\
n^{\prime}(x) & =n^{(0)}(x)+n^{(1)}(x)+\cdots .
\end{aligned}
$$

These series are obtained by successive substitution of (11) and (12), one into the other. For zero order (no recombination approximation), $J_{n}(x)=J_{n}{ }^{(0)}$. Substituting this in (11), we obtain the zero-order term of $n^{\prime}(x)$. This term, when substituted in (12), results in the first-order term of $J_{n}(x)$ and so forth:

$$
J_{n}{ }^{(0)}(x)=J_{n}{ }^{(0)}
$$

$$
\begin{aligned}
& n^{\prime(0)}(x)=-\frac{J_{n}{ }^{(0)}}{q N_{A}(x)} \int_{x}^{x_{2}} \frac{N_{A}(\bar{x})}{D_{n}} d \bar{x} \\
& J_{n}^{(1)}(x)=-\frac{1}{\tau_{n}} \cdot J_{n}{ }^{(0)} \int_{x_{1}}^{x} d \bar{x} \frac{1}{N_{A}(\bar{x})} \int_{\bar{x}}^{x_{2}} d \overline{\bar{x}} \frac{N_{A}(\overline{\bar{x}})}{D_{N}} .
\end{aligned}
$$

In particular,

$$
\begin{aligned}
n_{1}^{\prime} & \equiv n^{\prime(0)}\left(x_{1}\right)=-\frac{J_{n}{ }^{(0)}}{q N_{A}\left(x_{1}\right)} \cdot \int_{x_{1}}^{x_{2}} \frac{N_{A}(x)}{D_{n}} d x \\
J_{n}{ }^{(1)} & \equiv J_{n}^{(1)}\left(x_{2}\right) \\
& =-\frac{1}{\tau_{n}} J_{n}{ }^{(0)} \int_{x_{1}}^{x_{2}} d x \frac{1}{N_{A}(x)} \int_{x}^{x_{2}} d \bar{x} \frac{N_{A}(\bar{x})}{D_{n}}
\end{aligned}
$$

1 Further, we assume that the lifetime does not change along the base. This assumption is commonly used, and it allows simple expres-

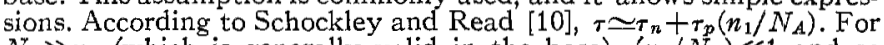
$N_{A} \gg n_{1}$ (which is generally valid in the base) $\left(n_{1} / N_{A}\right) \ll 1$ and so $\tau=\tau_{n}=\left(1 / K_{p} N_{t}\right)$, where $N_{t}$, the recombination centers concentration, and $K_{p}$, their capture cross section, do not vary in the base. Nevertheless, one can assume any kind of theoretical or empirical dependence of lifetime on impurity concentration and still apply the following procedure, if one keeps the lifetime inside the integral sign in all the following derivations. 
where (18) together with (4) yield the electron leakage current and enable (when the hold leakage current is also known) computation of emitter efficiency [2], and (19) is the total recombination current in the base up to first order in recombination:

$$
J_{n \boldsymbol{r}} \simeq J_{n}^{(1)} \text {. }
$$

Using (6), $\alpha_{T}$, to a first-order approximation is readily obtained:

$$
1-\alpha_{T}=-\frac{J_{n r}}{J_{n}(0)} \simeq-\frac{J_{n}{ }^{(1)}}{J_{n}(0)}
$$

and so also the base transit time $t_{\mathrm{tr}}$ :

$t_{t \mathrm{r}}=\int_{x_{1}}^{x_{2}} \frac{1}{v(x)} d x=q \int_{x_{1}}^{x_{2}} \frac{n^{\prime}(x)}{J_{n}(x)} d x \simeq-\tau_{n} \cdot \frac{J_{n}{ }^{(1)}}{J_{n}{ }^{(0)}}$.

Higher order approximations for $\alpha_{T}$ can be obtained by including higher order terms of (13).

\section{Calculations for Gaussian IMPURITY DISTRIBUTION}

In most common double-diffused transistor production processes, the assumption of a Gaussian distribution profile for acceptor impurity diffusion gives a very good approximation, especially in the base region. Therefore, we choose to apply the procedure described in Section II to the Gaussian-distribution case:

$$
N_{A}=N_{S} e^{-Z^{2}}
$$

where

$$
Z=\frac{x}{2 \lambda}, \quad \lambda=\sqrt{D_{A} t}
$$

and this distribution is assumed to exist between the normalized emitter and collector junction depths:

$$
Z_{1}=x_{1} / 2 \lambda \quad Z_{2}=x_{2} / 2 \lambda .
$$

We first assume

$$
D_{n}=\text { const }
$$

and by substituting (23) in (16), (18), and (19), we obtain

$$
\begin{aligned}
J_{n}{ }^{(0)} & =n_{1}{ }^{\prime} \cdot \frac{q D_{n}}{2 \lambda} \cdot \frac{1}{F\left(Z_{1}, Z_{2}\right)} \\
n^{\prime}(0) & (Z)=\frac{2 \lambda}{q D_{n}} J_{n}{ }^{(0)} \cdot F\left(Z, Z_{2}\right)=n_{1}{ }^{\prime} \cdot \frac{F\left(Z, Z_{2}\right)}{F\left(Z_{1}, Z_{2}\right)} \\
J_{n}{ }^{(1)} & =-\frac{(2 \lambda)^{2}}{L_{n}^{2}} \cdot J_{n}{ }^{(0)} \int_{z_{1}}^{z_{2}} F\left(Z, Z_{2}\right) d z
\end{aligned}
$$

where

$$
F\left(Z, Z_{2}\right)=\frac{\sqrt{\pi}}{2} e^{Z^{2}}\left(\operatorname{erf} Z_{2}-\operatorname{erf} Z\right) .
$$

With $u_{1}$ defined in (9), and using (21) and (22)

$$
\alpha_{T}=1-u_{1} \frac{W^{2}}{L_{n}^{2}}
$$

where

$$
\begin{aligned}
& t_{\mathrm{tr}}=u_{1} \cdot \frac{W^{2}}{D_{n}} \\
& u_{1}=\int_{Z_{1}}^{Z_{2}} F\left(Z, Z_{2}\right) d Z /\left(Z_{2}-Z_{1}\right)^{2} .
\end{aligned}
$$

The functions $F\left(Z, Z_{2}\right)$ and $u_{1}\left(Z_{1}, Z_{2}\right)$ were numerically evaluated and plotted, using the Tel-Aviv University CDC 6600 computer for various values of the parameter $Z_{2}$, and are presented in Figs. 2 and 3 . Observe that for case $Z_{1} \rightarrow Z_{2}$ or case $Z_{1}, Z_{2} \rightarrow 0$ (which corresponds to $\lambda \rightarrow \infty)$, we approach the homogeneous-base case and $u_{1} \rightarrow \frac{1}{2}$.

\section{Mobility Variation with Concentration}

For convenience in solving the excess minority carriers differential equation, the diffusion coefficiet $D_{n}$ has been generally taken to be some constant average value. Using the series expansion procedure of Section II, this imprecise approximation can be easily avoided by substituting $D_{n}$ 's dependence in $N_{A}$ into (16)-(19).

In this work we approximate the mobility (and hence the diffusivity) dependence on impurity concentration in $S_{i}$, by distinguishing three regions of behavior: for low and high doping, $D_{n}$ and $\mu_{n}$ are nearly constant, and in the intermediate region they are assumed to vary according to a power law.

The low and middle regions, which are of practical interest, are defined by

$$
\begin{array}{ll}
D=D_{0}, & N<N_{0} \\
D=D_{0}\left(\frac{N}{N_{0}}\right)^{-K}, & N_{0}<N<10^{19} \mathrm{~cm}^{-3} .
\end{array}
$$

By fitting the $\log -\log$ plot of mobility versus impurity concentration [4] by straight lines, and using Berry's value [5] for the power exponent, the following typical values for use in (34) and (35) are found.

\begin{tabular}{lccc}
\hline & Electrons & Holes & Unit \\
\hline$D_{0}$ & 32 & 12 & $\mathrm{~cm}^{2} / \mathrm{s}$ \\
$N_{0}$ & $5.10^{15}$ & $5.10^{15}$ & $\mathrm{~cm}^{-3}$ \\
$K$ & 0.27 & 0.27 & - \\
\hline
\end{tabular}

By analogy with Section III, using (34) and (35) in (16), (18), and (19), calculations for the Gaussian distribution (23) were done for the region of varying diffusivity (35):

$$
J_{i n}^{(0)}=n_{1}^{\prime} \cdot \frac{q D_{n}\left(x_{2}\right)}{2 \lambda} \cdot \frac{1}{F^{*}\left(Z_{1}, Z_{2}\right)}
$$

$$
\begin{aligned}
n^{\prime(0)}(Z) & =\frac{2 \lambda}{q D_{n}\left(x_{2}\right)} J_{n}{ }^{(0)} \cdot F^{*}\left(Z, Z_{2}\right)=n_{1}^{\prime} \cdot \frac{F^{*}\left(Z, Z_{2}\right)}{F^{*}\left(Z_{1}, Z_{2}\right)} \\
J_{n}{ }^{(1)} & =-\frac{(2 \lambda)^{2}}{L_{n}\left(x_{2}\right)^{2}} \cdot J_{n}{ }^{(0)} \int_{Z_{1}}^{Z_{2}} F^{*}\left(Z, Z_{2}\right) d Z
\end{aligned}
$$

where our choice to evaluate $D_{n}$ in $x_{2}$ is permitted by the freedom in defining $F^{*}$ : 


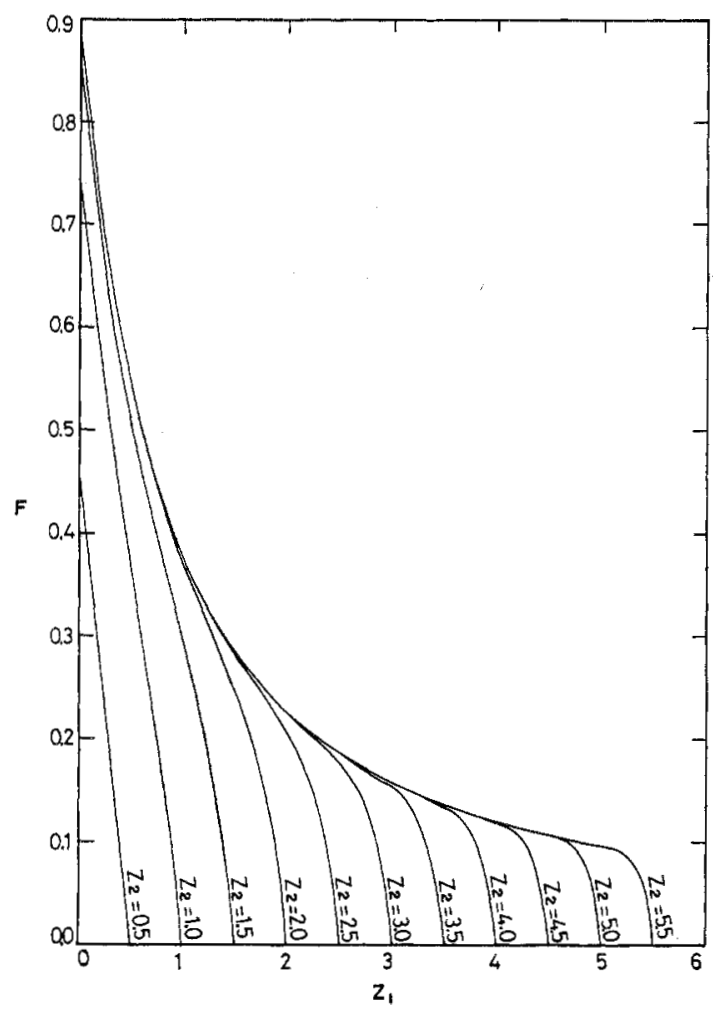

Fig. 2. Plots of $F\left(Z, Z_{2}\right)$ (Gaussian profile; $D_{n}=$ const).

$$
n^{\prime(0)}(Z)=n_{1}^{\prime} \cdot \frac{F\left(Z, Z_{2}\right)}{F\left(Z_{1}, Z_{2}\right)} .
$$

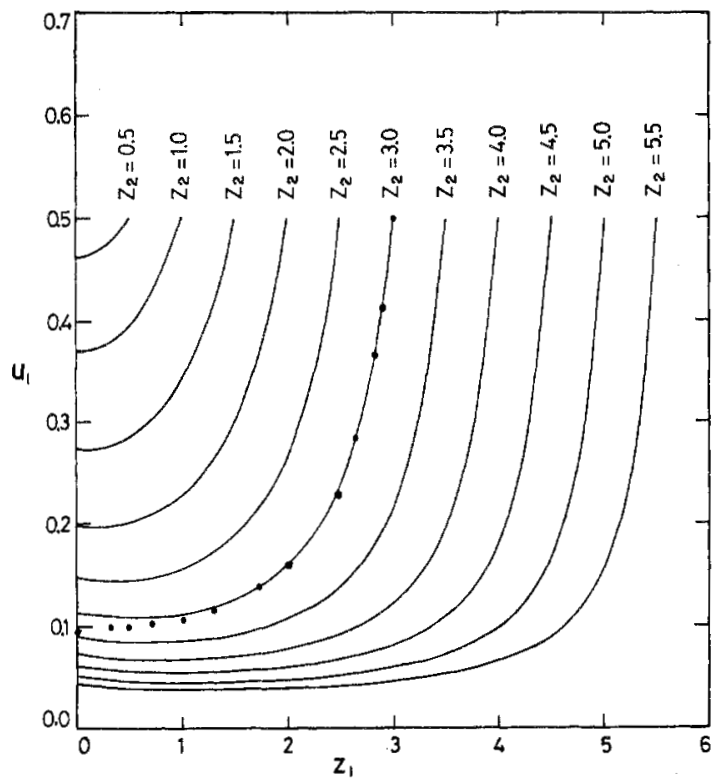

Fig. 3. Plots of $u_{1}\left(Z_{1}, Z_{2}\right)$ (Gaussian profile; $D_{n}=$ const).

$$
\alpha_{T}=1-u_{1} \cdot \frac{W^{2}}{L_{n}^{2}}, \quad t_{\mathrm{tr}}=u_{1} \cdot \frac{W^{2}}{D_{n}}, \quad \omega_{T}=\frac{\omega_{0}}{u_{1}} .
$$

The dots describe $u_{1}$ of exponential profile for comparison w ten $Z_{2}=3$.

$$
\begin{aligned}
F^{*}\left(Z, Z_{2}\right)=\frac{\sqrt{\pi}}{2 \sqrt{1+K}} e^{Z^{2}+K Z_{2}^{2}}\left[\operatorname{erf}\left(Z_{2} \sqrt{1+K}\right)\right. \\
-\operatorname{erf}(Z \sqrt{1+K})] .
\end{aligned}
$$

From (21), (22), and (38)

$$
\begin{aligned}
\alpha_{T} & =1-u_{1}{ }^{*} \frac{W^{2}}{L_{n}\left(x_{2}\right)^{2}} \\
t_{\mathrm{tr}} & =u_{1} * \frac{W^{2}}{D_{n}\left(x_{2}\right)}
\end{aligned}
$$

where

$$
u_{1}^{*}=\int_{Z_{2}}^{Z_{2}} F^{*}\left(Z, Z_{2}\right) d Z /\left(Z_{2}-Z_{1}\right)^{2} .
$$

Here again $F^{*}\left(Z, Z_{2}\right)$ and $u_{1}^{*}\left(Z_{1}, Z_{2}\right)$ were numerically calculated and plotted for various values of $Z_{2}$ and are presented in Figs. 4 and 5. As $K$ is the same for both $\mathrm{p}-\mathrm{n}-\mathrm{p}$ and $\mathrm{n}-\mathrm{p}-\mathrm{n}$ transistors, these graphs apply in both cases.

By comparing the plots for varying diffusivity with the plots for constant diffusivity, it is verified that the variation of diffusivity has an observable effect on transistor parameters. Referring to Fig. 5, we observe that $u_{1}{ }^{*}>0.3$ always and also happens to be greater than 0.5 , indicating the limited improvement in transit time and the $\alpha_{T}$ defect of the Gaussian-distributed-base drift transistor relative to the homogeneous-base transistor with base impurity concentration equal to that at the collector junction of the drift transistor.

\section{Application to Transistor Design}

A simple transformation of the parameter $u_{1}$ introduces a much more convenient parameter for design purpose:

$$
\begin{gathered}
v_{1}=u_{1} \cdot\left(Z_{2}-Z_{1}\right)^{2}=\int_{Z_{1}}^{Z_{2}} F\left(Z, Z_{2}\right) d Z \\
v_{2}^{*}=u_{1}^{*} \cdot\left(Z_{2}-Z_{1}\right)^{2}=\int_{Z_{1}}^{Z_{2}} F^{*}\left(Z, Z_{2}\right) d Z
\end{gathered}
$$

giving in turn

$$
\begin{aligned}
\alpha_{T} & =1-v_{1} \cdot \frac{(2 \lambda)^{2}}{L_{n}^{2}} \\
t_{\mathrm{tr}} & =v_{1} \cdot \frac{(2 \lambda)^{2}}{D_{n}}
\end{aligned}
$$

and in the case of varying diffusivity

$$
\begin{aligned}
\alpha_{T} & =1-v_{1}^{*} \cdot \frac{(2 \lambda)^{2}}{\left(L_{n}\left(x_{2}\right)\right)^{2}} \\
t_{\mathrm{tr}} & =v_{1}^{*} \cdot \frac{(2 \lambda)^{2}}{D_{n}\left(x_{2}\right)} .
\end{aligned}
$$

The parameters $v_{1}$ and $v_{1}^{*}$ are plotted in Figs. 6 and 7 . For given collector substrate doping and base im- 


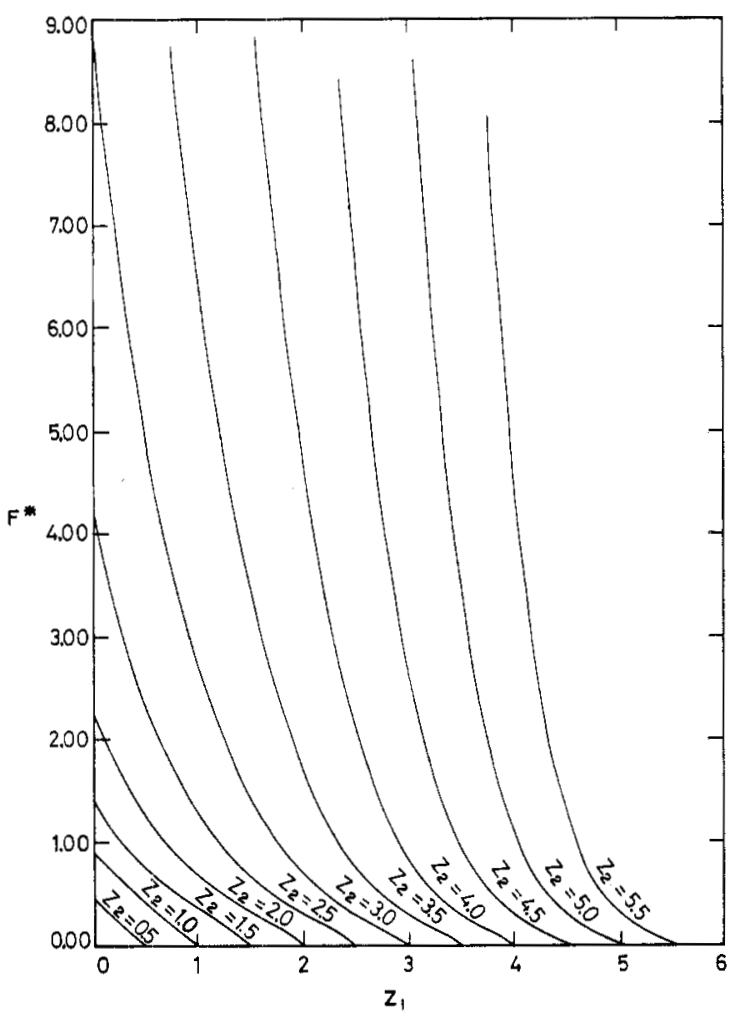

Fig. 4. Plots of $F^{*}\left(Z, Z_{2}\right)$ (Gaussian profile; $D_{n}=D_{0}\left(N / N_{0}\right)^{-0.27}$ ). $n^{\prime(0)}(Z)=n^{\prime} \frac{F^{*}\left(Z, Z_{2}\right)}{F\left(Z_{1}, Z_{2}\right)}$

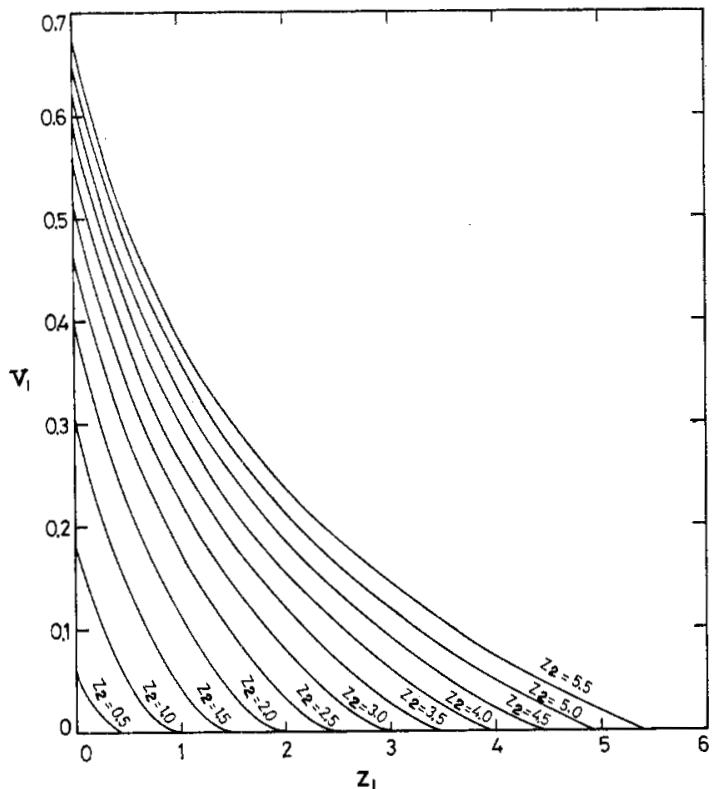

Fig. 6. Plots of $v_{1}\left(Z_{1}, Z_{2}\right)$ (Gaussian profile; $D_{n}=$ const).

$$
\alpha_{T}=1-v_{1} \cdot \frac{(2 \lambda)^{2}}{L_{n}^{2}}, \quad t_{\mathrm{tr}}=v_{1} \cdot \frac{(2 \lambda)^{2}}{D_{n}} \quad \omega T=\frac{1}{v_{1}} \cdot \frac{D_{n}}{(2 \lambda)^{2}} .
$$

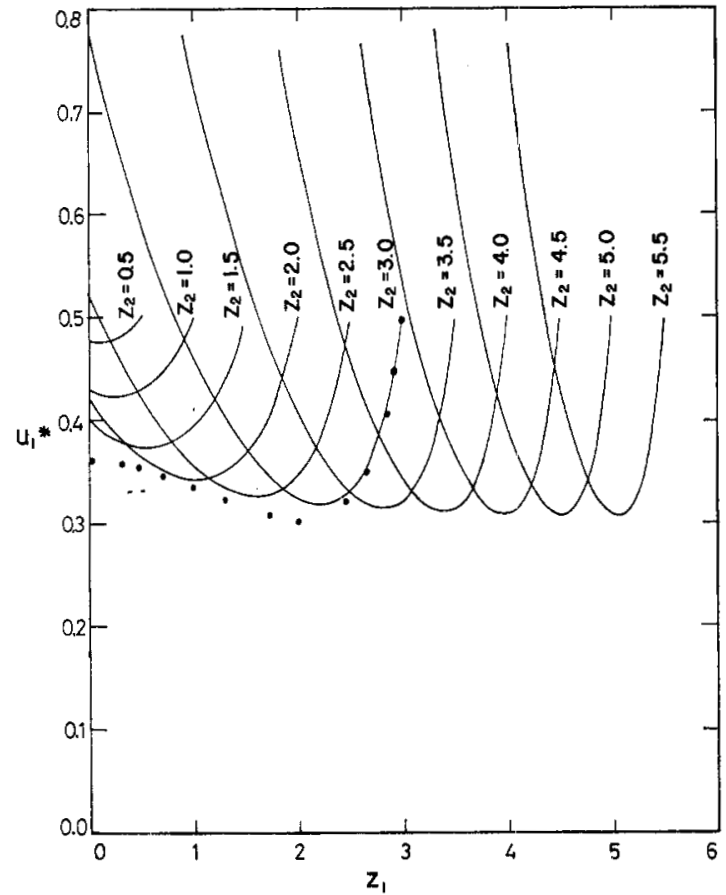

Fig. 5. Plots of $u_{1}^{*}\left(Z_{1}, Z_{2}\right)$ (Gaussian profile; $D_{n}=D_{0}\left(N / N_{0}\right)^{-0.27}$ ). $\alpha_{T}=1-u_{1}^{*} \cdot \frac{W^{2}}{L_{n}\left(x_{2}\right)^{2}}, \quad t_{\mathrm{tr}}=u_{1}^{*} \cdot \frac{W^{2}}{D_{n}\left(x_{2}\right)}, \quad \omega_{T}=\frac{\omega_{0}\left(x_{2}\right)}{u_{1}}$.

The dots describe $u_{1}$ * of exponential profile for comparison when $Z_{2}=3$.

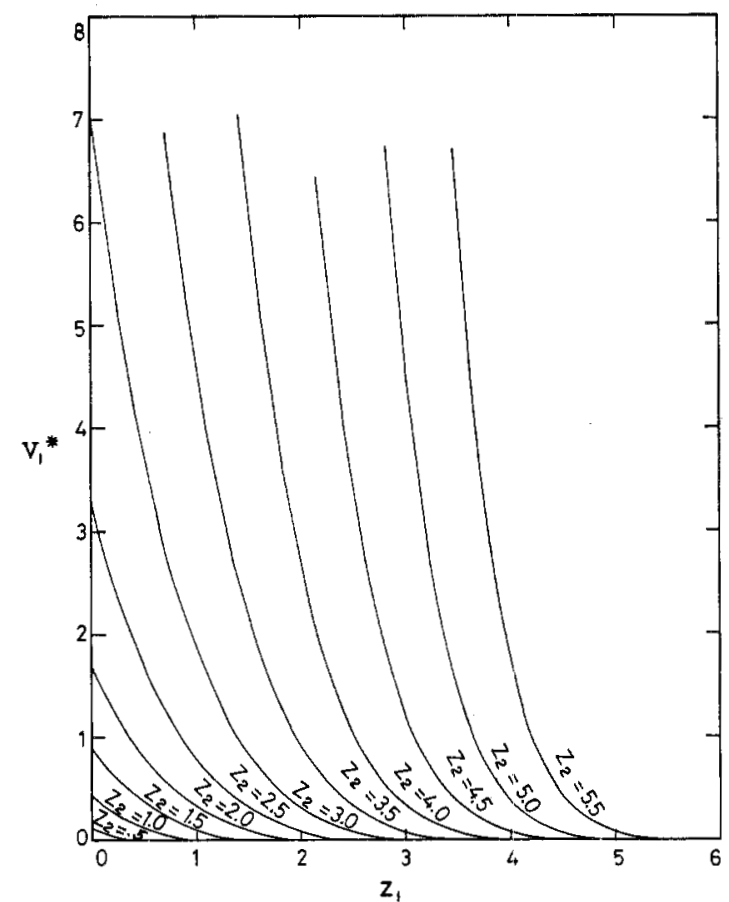

Fig. 7. Plots of $v_{1}^{*}\left(Z_{1}, Z_{2}\right)$ (Gaussian profile; $\left.D_{n}=D_{0}\left(N / N_{0}\right)^{-0.27}\right)$. $\alpha_{T}=1-v_{1}^{*} \cdot \frac{(2 \lambda)^{2}}{\left[L_{n}\left(x_{2}\right)\right]^{2}}, \quad t_{\mathrm{tr}}=v_{1}{ }^{*} \cdot \frac{(2 \lambda)^{2}}{D_{n}\left(x_{2}\right)}, \quad \omega_{T}=\frac{1}{v_{1}{ }^{*}} \frac{D_{n}\left(x_{2}\right)}{(2 \lambda)^{2}}$. 
purity diffusion conditions, $\lambda$ and $Z_{2}$ are determined ty (23)-(25). Thus as all dependence in $Z_{1}$ is included $n$ $v_{1}$ we can, using the plot of $v_{1}$ for the specified $z_{2}$, directly observe the variation of $\alpha_{T}$ and $t_{\mathrm{tr}}$ with emittar junction depth $Z_{1}$. On the other hand, for given emitter and base diffusion conditions, $\lambda$ and $Z_{1}$ are determined and we can examine the variation of parameters with collector substrate background concentration by movirıg vertically among the different plots of various $Z_{2}$ for the same specified $Z_{1}$.

It should be noted that each of the parameters $u_{1}, v_{1}$ and $u_{1}{ }^{*}, v_{1}{ }^{*}$ can be used only in the cases where the impurity concentration values lie in either the region of constant diffusivity (34) or in the region of varyir.g. diffusivity (35). Fortunately, base impurities concentration of a double-diffused transistor generally lies only in the region of varying diffusivity (35) [5].

In the case where base impurity concentration values lie in both $D_{n}$ regions (34) and (35), the integrals in (19) must be divided into three terms and the resultir gr parameter $u_{1_{\text {eff }}}$, which can be expressed in terms of the formerly computed parameters $v_{1}$ and $v_{1}^{*}$ :

$$
\begin{aligned}
& \alpha_{T}=1-u_{l_{\text {eff }}} \cdot \frac{W^{2}}{L_{n}\left(x_{2}\right)^{2}}=1-v_{1_{\text {eff }}} \cdot \frac{(2 \lambda)^{2}}{L_{n}\left(x_{2}\right)^{2}} \\
& t_{\mathrm{tr}}=u_{\mathrm{leff}} \cdot \frac{W^{2}}{D_{n}\left(x_{2}\right)}=v_{\mathrm{I}_{\mathrm{eff}}} \cdot \frac{(2 \lambda)^{2}}{D_{n}\left(x_{2}\right)} \\
& v_{1_{\text {gff }}}=v_{1}^{*}\left(Z_{1}, Z_{0}\right)+v_{1}\left(Z_{0}, Z_{2}\right)+\frac{\pi}{4}\left[\operatorname{erf} Z_{2}-\operatorname{erf} Z_{0}\right] \\
& \cdot\left[i \cdot \operatorname{erf}\left(i Z_{0}\right)-i \cdot \operatorname{erf}\left(i Z_{1}\right)\right] \\
& u_{1_{\text {eff }}}=v_{\mathrm{leff}_{\mathrm{eff}}} /\left(Z_{2}-Z_{1}\right)^{2}
\end{aligned}
$$

where $Z_{0}$ is defined by

$$
N\left(Z_{0}\right)=N_{0} .
$$

The parameters $v_{1}$ and $v_{1}{ }^{*}$ are plotted in Figs. 6 and ": and $\operatorname{erf} Z$ and $i \cdot \operatorname{erf}(i Z)$ are known tabulated function:

\section{Cutoff Frequencies and Excess Phase Shift}

For ac conditions, the continuity equation (2) be:comes

$$
\frac{\partial n^{\prime}}{\partial t}=\frac{1}{q} \frac{\partial J_{n}(x)}{\partial x}-\frac{n^{\prime}}{\tau_{n}} .
$$

Eliminating the time dependence, assuming $e^{i \omega t}$ variation,

$$
\frac{n^{\prime}}{\tau_{n} /\left(1+i \omega \tau_{n}\right)}=\frac{1}{q} \cdot \frac{d J_{n}}{d x} .
$$

Equation (55) is of the same form as (2), and the a: boundary conditions also resemble those of the dc cas: [4]; therefore, the dc solution may be used to find the ac parameters by simply substituting:

$$
\begin{aligned}
& \tau_{n} \rightarrow \tau_{n} /\left(1+i \omega \tau_{n}\right) \\
& L_{n} \rightarrow L_{n} /\left(1+i \omega \tau_{n}\right)^{\frac{1}{2}} .
\end{aligned}
$$

In place of (9) we now have

$$
\begin{array}{r}
\alpha_{T}=1-u_{1} \cdot \frac{W^{2}}{L_{n}{ }^{2}}\left(1+i \omega \tau_{n}\right)+u_{2} \cdot \frac{W^{4}}{L_{n}{ }^{4}}\left(1+i \omega \tau_{n}\right)^{2} \\
-\ldots .
\end{array}
$$

This discussion is concerned with high $\beta$ transistors. For the practical region of frequencies

$$
\left(1-\alpha_{T}\right)_{\mathrm{do}} \ll \frac{\omega}{\omega_{0}} \ll 1
$$

the following expansion of $\alpha_{T}$ results in

$$
\alpha_{T}=1-u_{1} \cdot\left(\frac{i \omega}{\omega_{0}}\right)+u_{2} \cdot\left(\frac{i \omega}{\omega_{0}}\right)^{2}-\cdots
$$

where $\omega_{0}$ is defined by

$$
\omega_{0}=\frac{D_{n}\left(x_{2}\right)}{W^{2}} .
$$

We have, therefore, the interesting result that the same series expansion coefficients developed in Section II for $\alpha_{T}$ in terms of recombination apply also for expansion of $\alpha_{T}$ in terms of frequency.

A common approximation for $\alpha$, dependence on frequency, is the single pole with excess phase shift

$$
\alpha_{T}=\frac{\alpha_{0} e^{-i m \omega / \omega_{\alpha}}}{1+i \frac{\omega}{\omega_{\alpha}}}
$$

which when substituted in the definition for $\beta$ gives (using first-order approximation in the denominator)

$$
\begin{aligned}
& \beta_{T}=\frac{\beta_{0} e^{-i m \omega / \omega_{\alpha}}}{1+i \frac{\omega}{\omega_{\beta}}}=\frac{e^{-i m \omega / \omega_{\alpha}}}{1 / \beta_{0}+i \frac{\omega}{\omega_{T}}} \\
& \omega_{T}=\omega_{\beta} \cdot \beta_{0}=\frac{\omega_{\alpha}}{1+\alpha_{0} \cdot m} .
\end{aligned}
$$

There are several ways to fit approximation (61) to the real solution for $\left(\alpha_{T}\right)_{\mathbf{s c}}$, and thus define the parameters $\omega_{\alpha}, \omega_{\beta}, \omega_{T}, m$. For some reasons it is preferable to have a good fit for the region $\omega \ll \omega_{\alpha}$ instead of the region around $\omega_{\alpha}$. Accordingly, Gummel [6] proposed a lowfrequency definition of $\omega_{T}$ :

$$
\frac{1}{\omega_{T}}=\left.\frac{d}{d \omega} I_{m}\left(\frac{1}{\beta}\right)\right|_{\omega \rightarrow 0}
$$

This is effectively a first-order approximation of $\beta(\omega)$ according to (62). Regarding (59), we observe that the imaginary part of $\alpha_{T}$ in the low-frequency limit is really the first-order term of the $\alpha_{T}$ expansion in fre- 
quency. Therefore, the following definition is equivalent to Grummel's definitions:

$$
\frac{1}{\omega_{T}}=-\left.\frac{d \alpha(\omega)}{d(i \omega)}\right|_{\omega \rightarrow 0}
$$

or using (59)

$$
\omega_{T}=\frac{\omega_{0}}{u_{1}}
$$

and further

$$
\omega_{\beta}=\frac{\omega_{0}}{\beta_{0} u_{1}}
$$

with $\omega_{0}$ defined in $(60)$.

Note that (32) and (66) give the relation $\omega_{T}=1 / t_{\mathrm{tr}}$, as would be expected.

Our suggestion is to extend Gummel's approach in order also to define $\omega_{\alpha}$ and $m$. As explained in the Appendix, these parameters cannot be computed by firstorder fitting. Therefore, we do a low-frequency fit of (61) to (59) up to second order and obtain the equations

$$
\begin{aligned}
& \frac{u_{1}}{\omega_{0}}=\frac{m+1}{\omega_{\alpha}}\left(=\frac{1}{\omega_{T}}\right) \\
& \frac{u_{2}}{\omega_{0}^{2}}=\frac{1}{2} \frac{m^{2}+2 m+2}{\omega_{\alpha}^{2}} .
\end{aligned}
$$

Solving (68) and (69) simultaneously, we get

$$
\begin{aligned}
m & =\frac{1}{\sqrt{2 P-1}}-1 \\
\omega_{\alpha} & =\frac{\omega_{0}}{u_{1} \cdot \sqrt{2 P-1}}\left[=\omega_{0} \cdot \frac{m+1}{u_{1}}\right]
\end{aligned}
$$

where

$$
P \equiv \frac{u_{2}}{\left(u_{1}\right)^{2}}
$$

and $u_{1}$ and $u_{2}$ result from the general procedure of Section II :

$$
\begin{gathered}
u_{1}=\frac{D\left(x_{2}\right)}{W^{2}} \int_{x_{1}}^{x_{2}} d x \frac{1}{N_{A}(x)} \int_{x}^{x_{2}} d \bar{x} \frac{N_{A}(\bar{x})}{D_{n}} \\
u_{2}=-\frac{D\left(x_{2}\right)^{2}}{W^{4}} \int_{x_{1}}^{x_{2}} d x \frac{1}{N_{A}} \int_{x}^{x_{2}} d \bar{x} \frac{N_{A}}{D_{n}} \int_{x_{1}}^{\bar{x}} d \overline{\bar{x}} \\
\cdot \frac{1}{N_{A}} \int_{\overline{\bar{x}}}^{x_{2}} d \bar{x} \frac{N_{A}}{D_{n}}
\end{gathered}
$$

One may compute $u_{1}$ and $u_{2}$ numerically for general impurity distribution $N_{A}(x)$ and with any assumption about $D_{n}$. Having these two parameters, we have shown how all $\mathrm{dc}$ and ac base gain parameters can be readily computed.

\section{Homogeneous-Base ExAMple}

In order to test the expressions developed in Section VI, we apply them to the homogeneous-base situation. For this case, the exact solution is known [1] and the coefficients $u_{1}$ and $u_{2}$ are obtained by expanding it:

$$
\alpha_{T}=\operatorname{sech} \frac{W}{L_{n}}=1-\frac{1}{2} \cdot \frac{W^{2}}{L_{n}^{2}}+\frac{5}{24} \cdot \frac{W^{4}}{L_{n}^{4}}
$$

so that

$$
\begin{aligned}
& u_{1}=\frac{1}{2} \\
& u_{2}=\frac{5}{24}
\end{aligned}
$$

and

$$
p=\frac{u_{2}}{u_{1}^{2}}=\frac{5}{6} .
$$

Using (66), (67), (70), and (71)

$$
\begin{aligned}
& \omega_{T}=\frac{u_{0}}{u_{1}}=2 \frac{W^{2}}{D_{n}} \\
& \omega_{\beta}=\frac{\omega_{0}}{\beta u_{1}}=2 \frac{W^{2}}{\beta_{0} D_{n}} \\
& m=\frac{1}{\sqrt{2 p-1}}-1=\sqrt{\frac{3}{2}-1=0.22} \\
& \omega_{\alpha}=\frac{\omega_{0}}{u_{1} \sqrt{2 p-1}}=\sqrt{6} \frac{W^{2}}{D_{n}}=2.45 \frac{W^{2}}{D_{n}}
\end{aligned}
$$

in good agreement with classical results [4].

\section{ViII. Exponential Distribution Example}

Equations (73) and (74) are here applied to the exponential distribution for both constant and power-lawdependent diffusion coefficient:

$$
N_{A}(x)=N_{A_{E B}} e^{-\eta x / w}
$$

where $x=0$ is now the emitter-base junction.

For constant $D_{n}$, integration of (73) and (74) yields the expression

$$
u_{1}=\frac{\eta+e^{-\eta}-1}{\eta^{2}}
$$

which is identical to that given by the conventional treatment [4], and the expression

$$
u_{2}=\frac{\frac{1}{2} \eta^{2}-2+3 \eta e^{-\eta}+e^{-\eta}+e^{-2 \eta}}{\eta^{4}} .
$$

Equations (70) and (72) together with (84) and (85) give a new analytical expression for $m$. The functions $u_{1}$ and $m$ are plotted versus $\eta$ in the dotted lines in Figs. 8 and 9 , respectively. Both are in good agreement with 


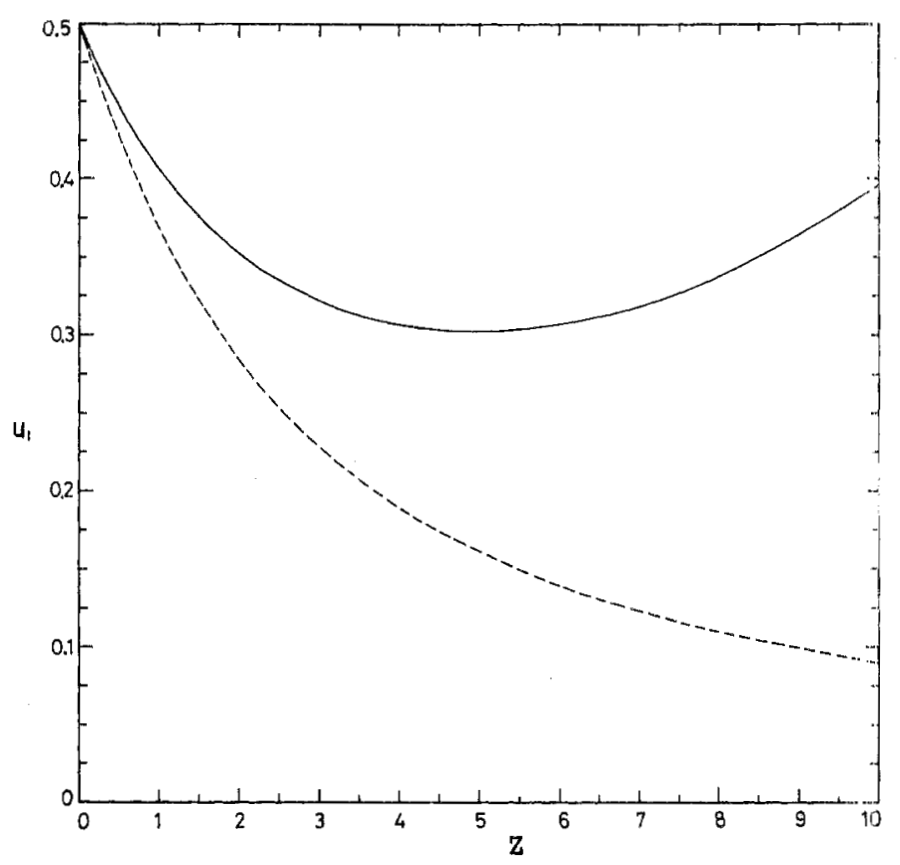

Fig. 8. Plots of $u_{1}$ and $u_{1}{ }^{*}$ (exponential profile; $u_{1}$, dotted lite: $D_{n}=$ const; $u_{1}{ }^{*}$, continuous line: $\left.D_{n}=D_{0}\left(N / N_{0}\right)^{-0.27}\right)$.

$$
\alpha_{T}=1-u_{1}^{*} \frac{W^{2}}{L_{n}\left(x_{2}\right)}, \quad t_{\mathrm{tr}}=u_{1}^{*} \cdot \frac{W^{2}}{D_{n}\left(x_{2}\right)}, \quad \omega_{1}=\frac{\omega_{0}\left(x_{2}\right)}{u_{1}^{*}}
$$

and correspondingly for $u_{1}$.

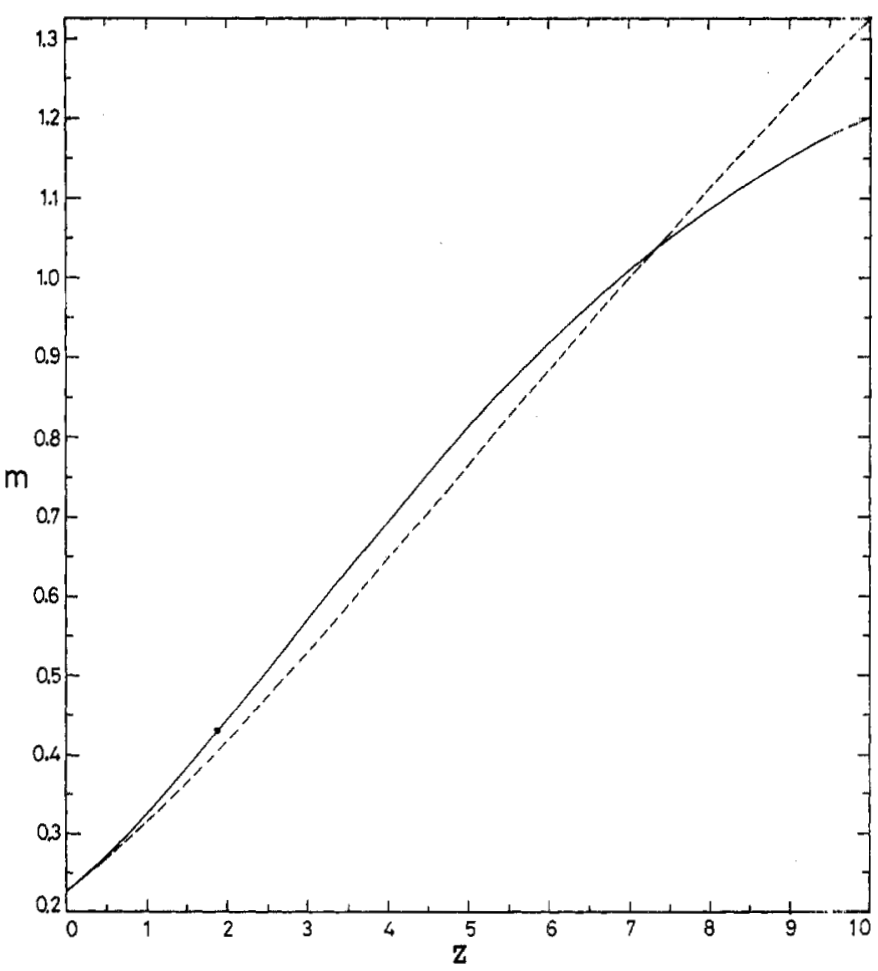

Fig. 9. Plots of $m$ and $m^{*}$ (exponential profile; $m$, dotted ine: $D_{n}=$ const; $m^{*}$, continuous line: $\left.D_{n}=D_{0}\left(N / N_{0}\right)^{-0.27}\right)$.

$$
\omega_{\alpha}=\frac{\omega_{T}}{m+1} .
$$

Kelly and Ghausi's plots derived using the method of: infinite product expansion of $\alpha$ in terms of its poles [8]. In the case of $\eta=4$, we obtain $m=0.645$ and $\omega_{\alpha} / \omega_{0}=m+1 / u_{1}=8.7$, whereas Kelly and Ghausi [8] give $m=0.62$ and $\omega_{\alpha} / \omega_{0}=8.1$. Our results fit better with Abraham's [7] results: $m=0.645$ and $\omega_{\alpha} / \omega_{0}=8.4$, calculated by fitting (61) to the exact computer calculation of $\alpha(\omega)$.

An advantage of our method is the possibility of calculating the parameters for the assumption of varying diffusion coefficient, which cannot be done in the two other methods.

Using (35) and (83) in (73) and (74),

$$
\begin{aligned}
u_{1}^{*}= & \frac{e^{K n}+K e^{-\eta}-(1+K)}{K(1+K) \eta^{2}} \\
u_{2}^{*}= & \frac{1}{\eta^{4}}\left\{\frac{1-2 K}{2 K^{2}(1-K)}-\frac{3+4 K}{K(1+K)(1+2 K)} e^{-\eta}\right. \\
& +\frac{1}{(1+K)} e^{-2 \eta}-\frac{1}{K^{2}(1+K)} e^{K \eta} \\
& +\frac{1+3 K}{2 K^{2}(1+K)^{2}(1+2 K)} \cdot e^{2 K \eta} \\
& \left.+\frac{3-K}{K(1+K)(1-K)} e^{(K-1) \eta}\right\} .
\end{aligned}
$$

Now $m^{*}$ is calculated from these parameters using (70). The functions $u_{1}{ }^{*}$ and $m^{*}$ were calculated assuming $K=0.27$ and are plotted versus $\eta$ in Figs. 8 and 9, respectively, in a continuous line. They are observably different from the corresponding parameters for constant diffusion coefficient.

It is also possible now to estimate the error of using exponential approximation instead of Gaussian distribution in the calculation of $u_{1}$ and $u_{1}{ }^{*}$. Using (23)-(25) and (83),

$$
\eta=\ln \frac{N_{A}\left(Z_{1}\right)}{N_{A}\left(Z_{2}\right)}=Z_{2}^{2}-Z_{1}^{2}
$$

which allows expressions (84) and (86) to be written as functions of $Z_{1}$ with $Z_{2}$ as parameters. The dots in Figs. 4 and 5 represent the calculation of these functions for $Z_{2}=3$ and various values of $Z_{1}$. This example illustrates the difference between the two distribution assumptions, indicating that the difference is not prominent in the case of constant $D_{n}$ (Fig. 4), but quite prominent for varying $D_{n}$ (Fig. 5 ). In both cases the difference decreases for increased $Z_{1}$.

\section{ApPENDix}

Following Abraham [7], we explain by means of a complex vector diagram (Fig. 10) why $\omega_{\beta}$ and $\omega_{T^{\prime}}$ can be defined from a first-order approximation of (59), while 


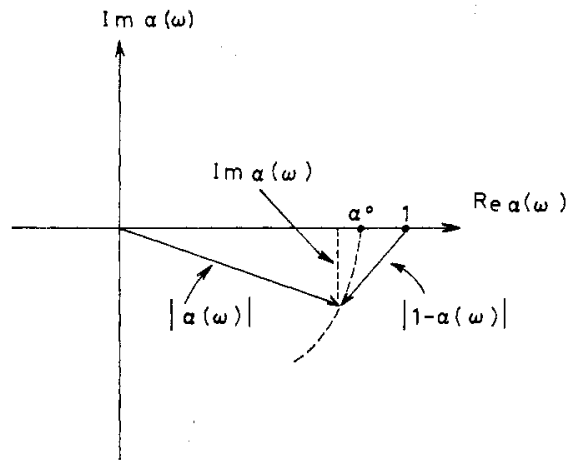

Fig. 10. $\alpha(\omega)$ in complex plane.

$\omega_{\alpha}$ and $m$ can be defined only by a second-order approximation.

At low frequencies the module of $\alpha(\omega)$ is approximately constant, but the module of $1-\alpha(\omega)$ may increase appreciably when

$$
I_{m} \alpha(\omega) \sim 1-\alpha_{0}
$$

As $\beta=\alpha / 1-\alpha,|\beta|$ rolloff is therefore expected to begin in this frequency range.

Regarding (58) or (59), we observe that for high $\beta$ transistors and for $\omega / \omega_{0} \ll 1$ (as is the case in the $\omega_{\beta}$ order of magnitude), $I_{m} \alpha(\omega)$ is approximated by the firstorder term of (59). Therefore, first-order effect explains $\beta$ rolloff and enables us to define $\omega_{\beta}$ [observe, moreover, substitution of (59) into (89) results in (67)].
First-order approximation cannot explain $|\alpha|$ rolloff. On the contrary, (59) up to first order will produce $\left|\alpha_{T}\right|>1$; it is only the second-order term that covers $\alpha$ rolloff through a decrease in the real part of $\alpha$.

\section{ACKNOWLEDGMENT}

The authors wish to acknowledge the assistance of Dr. D. H. Friedman in the final correction of this manuscript.

\section{REFERENCES}

[1] W. Shockley, "The theory of p-n junctions in semiconductors and p-n junction transistor," Bell Syst. Tech. J., vol. 28, pp. 435-489, July 1949

[2] J. L. Moll and I. M. Ross, "The dependence of transistor parameters on the distribution of base layer resistivity," Proc. IRE, vol. 44, pp. 72-78, Jan. 1956.

[3] H. Kroemer, "On diffusion and drift transistor theory," Arch. Elek. Übertragung, vol. 8, pp. 223-228, 1954.

[4] S. K. Ghandhi, The Theory and Practice of Microelectronics. New York: Wiley, 1968, ch. 14.

[5] R. Berry, "Correlation of diffusion process variations with varitions in electrical parameters of bipolat transistors," Proc. IEEE, vol. 57 , pp. 1513-1517, Sept. 1969

[6] H. K. Gummel, "On the definition of cutoff frequency $f_{T}$," Proc. IEEE (Lett.), vol. 57, p. 2159, Dec. 1969.

[7] R. P. Abraham, "Transistor behavior at high frequencies," IRE Trans. Electron Devices, vol. ED-7, pp. 59-69, Jan. 1960.

[8] J. J. Kelly and M.S. Ghausi, "Poles of the alpha of drift transistors," IEEE Trans. Circuit Theory, vol. CT-12, pp. 593-595, Dec. 1965.

[9] T.-C. Lo, "Base transport factor calculations for transistors with complementary error function and Graussian base doping profiles," IEEE Trans. Electron Devices, vol. ED-18, pp. 243248, Apr. 1971

[10] W. Shockley and W. T. Read, Jr., "Statistics of the recombination of holes and electrons," Phys. Rev., vol. 87, p. 855, Sept. 1952.

\title{
Numerical Investigation of the Thyristor Forward Characteristic
}

\author{
JOZEF CORNU, MEMBER, IEEE, AND MANFRED LIETZ
}

\begin{abstract}
Analytical solutions for the forward characteristic of thyristors have been limited to abrupt and constant doping profiles. Average values for the mobilities and the carrier lifetime had to be assumed for each region of the device.

Results will be presented here which are based on an exact numerical solution of the transport, continuity, and Poisson equations for the one-dimensional thyristor. Doping, mobility, and lifetime can be varied from point to point. Thyristor structures are much wider and higher doped than the devices treated numerically in the literature. The dependency of the forward characteristic on various device
\end{abstract}

Manuscript received December 13, 1971; revised March 22, 1972. This paper was presented at the IEEE Conference on Electron Devices, Washington, D. C., October 12, 1971.

The authors are with the Brown Boveri Research Center, $\mathrm{CH}$ 5401 Baden, Switzerland. parameters was examined, and the important results were verified experimentally.

For high current and a base width that is large compared to the diffusion length, the dependency of mobility on the carrier concentration leads to a base voltage drop significantly lower than that expected from analytical theory. Furthermore, it is shown that for this case the parameters of the highly doped side regions (doping, width, and lifetime) have much less influence than predicted from Herlet's [3] theory.

\section{INTRODUCTION}

$\pi$ $\checkmark H E$ operation of a $\mathrm{p}^{+}-\mathrm{n}-\mathrm{p}-\mathrm{n}^{+}$thyristor can be divided into four well-distinguishable states: turn-on, forward conduction, turn-off, and block- 